Jie Luo*, Guang-Na Gu and Ying-Li Zheng

\title{
Crystal structure of poly[diaqua- $\left(\mathrm{m}_{3}-3^{\prime}, 5^{\prime}-\right.$ dicarboxy-[1,1'-biphenyl]-3,4-dicarboxylato- $\left.K^{4} O, O^{\prime}: O^{\prime \prime}: O^{\prime \prime \prime}\right)$ cadmium(II)], $\mathrm{C}_{16} \mathrm{H}_{11} \mathrm{O}_{10} \mathrm{Cd}$
}

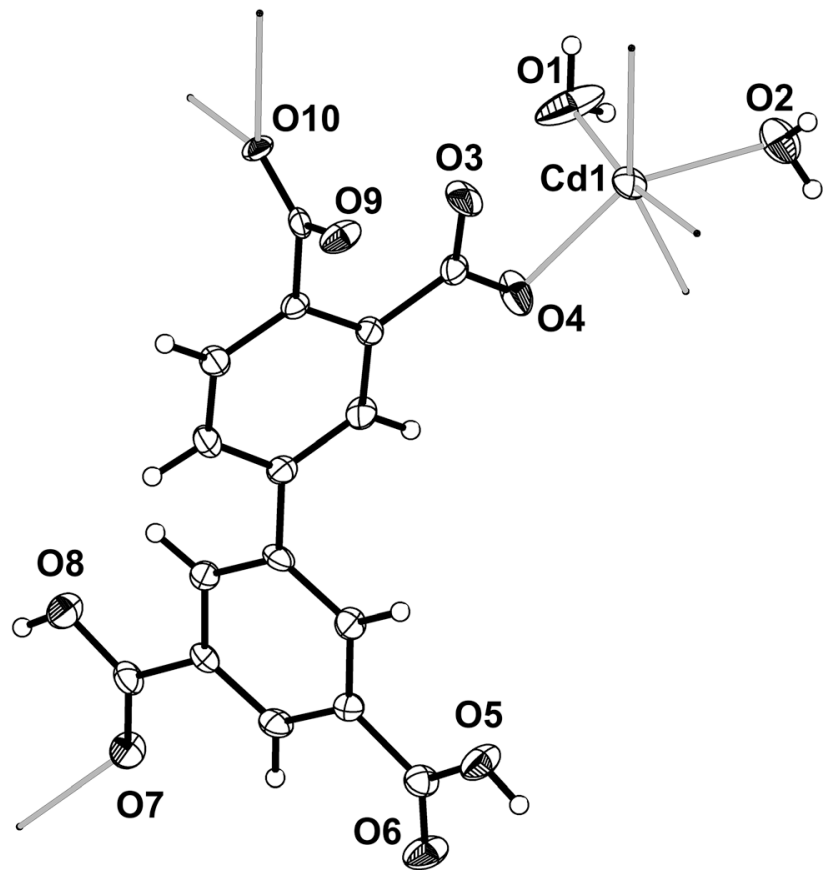

https://doi.org/10.1515/ncrs-2020-0420

Published online October 15, 2020

\section{Abstract}

$\mathrm{C}_{16} \mathrm{H}_{11} \mathrm{O}_{10} \mathrm{Cd}$, monoclinic, $C 2 / c$ (no. 15), $a=26.026(3) \AA$, $b=6.3807(6) \AA \AA, c=19.738(5) \AA, \alpha=90, \beta=98.162(16)$, $\gamma=90, V=3244.5(10) \AA^{3}, Z=8, R_{g t}(F)=0.0359$, $w R_{\text {ref }}\left(F^{2}\right)=0.0702, \mathrm{~T}=293(2) \mathrm{K}$.

\section{CCDC no.: 2032713}

Table 1 contains crystallographic data and Table 2 contains the list of the atoms including atomic coordinates and displacement parameters.

\footnotetext{
*Corresponding author: Jie Luo, School of Chemical Engineering and Pharmaceutics, Henan University of Science and Technology, Luoyang 471023, PR China, E-mail: luoj2020@126.com

Guang-Na Gu and Ying-Li Zheng, School of Chemical Engineering and Pharmaceutics, Henan University of Science and Technology, Luoyang 471023, PR China
}

Table 1: Data collection and handling.

\begin{tabular}{ll}
\hline Crystal: & Colourless block \\
Size: & $0.45 \times 0.35 \times 0.26 \mathrm{~mm}$ \\
Wavelength: & Mo $K \alpha$ radiation $(0.71073 \AA)$ \\
$\mu:$ & $1.40 \mathrm{~mm}^{-1}$ \\
Diffractometer, scan mode: & SuperNova, $\omega$ \\
$\theta_{\text {max }}$, completeness: & $25.5^{\circ},>99 \%$ \\
$N(h k l)_{\text {measured }}, N(h k l)_{\text {unique }}, R_{\text {int }}:$ & $9282,3010,0.036$ \\
Criterion for $I_{\text {obs }}, N(h k l)_{\text {gt }}:$ & $I_{\text {obs }}>2 \sigma\left(I_{\text {obs }}\right), 2521$ \\
$N(\text { param })_{\text {refined }}$ & 247 \\
Programs: & CrysAlis
\end{tabular}

\section{Source of material}

A mixture of $\mathrm{Cd}\left(\mathrm{NO}_{3}\right)_{2} \cdot 2 \mathrm{H}_{2} \mathrm{O}(0.1 \mathrm{mmol}, 0.0272 \mathrm{~g})$, and 3,3',4,5'-biphenyl tetracarboxylic acid (H4btc) ( $0.1 \mathrm{mmol}$, $0.0330 \mathrm{~g}$ ), was dissolved in $10 \mathrm{~mL} \mathrm{H}_{2} \mathrm{O}$. Then the solution was heated in a $25 \mathrm{~mL}$ Teflon-lined autoclave under autogenous pressure at $443 \mathrm{~K}$ for 4 days. After cooling to room temperature, crystals were collected.

\section{Experimental details}

Coordinates of hydrogen atoms were positioned based on the molecular restrictions. Their $U_{\text {iso }}$ values were set to $1.2 U_{e q}$ of the parent atoms.

\section{Comment}

In the last two decades, a large number of metal-organic frameworks (MOFs) have been reported. Organic aromatic multicarboxylates like the biphenyl-tetracates can yield predetermined networks and have been widely utilized to construct MOFs [4-8]. On the other hand, $3,3^{\prime}, 4,5^{\prime}$-biphenyl tetracarboxylic acid serves as bridging ligand $[9,10]$.

In the title complex, there are one $\mathrm{Cd}(\mathrm{II})$, two water molecules and one H2btc ligand in the asymmetric unit. 
Table 2: Fractional atomic coordinates and isotropic or equivalent isotropic displacement parameters $\left(\AA^{2}\right)$

\begin{tabular}{|c|c|c|c|c|}
\hline Atom & $x$ & $y$ & $z$ & $U_{\text {iso }}{ }^{*} / U_{\text {eq }}$ \\
\hline Cd1 & $0.71693(2)$ & $0.28960(4)$ & $0.41166(2)$ & $0.02573(11)$ \\
\hline 01 & $0.74533(14)$ & $0.4337(7)$ & $0.31824(16)$ & $0.0827(14)$ \\
\hline H11 & 0.742673 & 0.344302 & 0.285234 & $0.124^{\star}$ \\
\hline $\mathrm{H} 12$ & 0.778199 & 0.456398 & 0.327242 & $0.124^{\star}$ \\
\hline $\mathrm{O} 2$ & $0.75421(12)$ & $-0.0259(4)$ & 0.39677 (13) & $0.0400(8)$ \\
\hline H21 & 0.730647 & -0.120067 & 0.385414 & $0.060^{\star}$ \\
\hline 22 & 0.770153 & -0.070314 & 0.435515 & $0.060^{\star}$ \\
\hline 03 & 0.69635 (11) & $0.6598(4)$ & $0.47673(14)$ & $0.0364(7)$ \\
\hline 04 & $0.64513(12)$ & $0.4898(4)$ & $0.39848(14)$ & $0.0416(8)$ \\
\hline 05 & $0.38858(11)$ & $0.3694(4)$ & 0.39040 (13) & $0.0361(7)$ \\
\hline H5 & 0.370225 & 0.266998 & & $0.054^{\star}$ \\
\hline 06 & $0.32767(11)$ & $0.4328(4)$ & $0.30154(13)$ & $0.0382(8)$ \\
\hline 07 & $0.34180(11)$ & $1.1026(4)$ & $0.17462(13)$ & $0.0351(7)$ \\
\hline 08 & $0.41226(12)$ & $1.3050(4)$ & $0.20173(14)$ & $0.0334(7)$ \\
\hline $\mathrm{H} 8$ & $0.397(2)$ & $1.386(8)$ & $0.174(3)$ & $0.077(19)^{\star}$ \\
\hline 09 & $0.67154(11)$ & $7(4)$ & $0.59051(12)$ & $0.0299(7)$ \\
\hline 010 & $0.70490(10)$ & $1.1428(4)$ & $0.51627(12)$ & $0.0224(6)$ \\
\hline $\mathrm{C} 1$ & $0.61267(14)$ & $0.8043(5)$ & $0.44149(17)$ & $0.0191(8)$ \\
\hline $\mathrm{C} 2$ & 0.56459 (15) & $0.7792(6)$ & 0.40077 (17) & $0.0236(9)$ \\
\hline $\mathrm{H} 2 \mathrm{~A}$ & 0.558460 & 0.657899 & 0.3 & $0.028^{\star}$ \\
\hline C3 & 0.52559 (15) & $0.9292(5)$ & $0.39780(17)$ & $0.0211(9)$ \\
\hline $\mathrm{C} 4$ & $0.53595(15)$ & $1.1127(6)$ & $0.43528(18)$ & $0.0257(9)$ \\
\hline H4 & & & & $0.031^{\star}$ \\
\hline C5 & $0.58311(15)$ & $1.1392(6)$ & $0.47691(18)$ & $0.0240(9)$ \\
\hline H5A & 0.589089 & 1.261300 & 0.502528 & $0.029^{\star}$ \\
\hline C6 & $0.62161(14)$ & $0.9869(5)$ & $0.48111(16)$ & $0.0178(8)$ \\
\hline $\mathrm{C} 7$ & $0.47467(14)$ & $0.8905(6)$ & $0.35460(17)$ & $0.0213(8)$ \\
\hline C8 & $0.44744(15)$ & $0.7072(6)$ & $0.36300(17)$ & $0.0233(9)$ \\
\hline H8A & 0.461445 & 0.609221 & 0.395326 & $0.028^{\star}$ \\
\hline C9 & $0.39956(15)$ & $0.6676(5)$ & $0.32390(17)$ & $0.0215(9)$ \\
\hline C10 & $0.37941(15)$ & $0.8097(5)$ & $0.27365(17)$ & $0.0237(9)$ \\
\hline $\mathrm{H} 10 \mathrm{~A}$ & 0.347839 & 0.782562 & 0.246650 & $0.028^{*}$ \\
\hline C11 & $0.40640(15)$ & $0.9915(5)$ & $0.26384(17)$ & $0.0208(8)$ \\
\hline C12 & $0.45358(15)$ & $1.0346(6)$ & 0.30515 (17) & $0.0219(9)$ \\
\hline $\mathrm{H} 12 \mathrm{~A}$ & 0.470881 & & 0.299606 & $0.026^{*}$ \\
\hline C13 & $0.65441(15)$ & $0.6435(5)$ & $0.43950(17)$ & $0.0212(9)$ \\
\hline C14 & $0.36838(16)$ & $0.4795(6)$ & $0.33680(18)$ & $0.0253(9)$ \\
\hline C15 & $0.38340(16)$ & $1.1384(6)$ & $0.20916(18)$ & $0.0247(9)$ \\
\hline C16 & $0.67032(15)$ & $1.0219(5)$ & $0.53241(17)$ & $0.0198(9)$ \\
\hline
\end{tabular}

The cadmium atom is six-coordinated by two water ligands, and four oxygen atoms from four H2btc ligands. The $\mathrm{Cd}-\mathrm{O}$ bond lengths are all in the expected ranges.
Author contribution: All the authors have accepted responsibility for the entire content of this submitted manuscript and approved submission.

Funding information: The authors thank the Key Scientific Research Project for Universities of Henan Province (No. 20A430012) and $\mathrm{PhD}$ research startup foundation of Henan University of Science and Technology (No. 13480070) for financial support.

Conflict of interest statement: The authors declare no conflicts of interest regarding this article.

\section{References}

1. Agilent Technologies. CrysAlis ${ }^{\mathrm{PRO}}$ Software System, (Version 1.171.35.15); Agilent Technologies UK Ltd: Oxford, UK, 2011.

2. Sheldrick G. M. SHELXT - integrated space-group and crystalstructure determination. Acta Crystallogr. 2015, A71, 3-8.

3. Sheldrick G. M. Crystal structure refinement with SHELXL. Acta Crystallogr. 2015, C71, 3-8.

4. Li S. H., Zang J., Guo J. B. Synthesis and crystal structure of a new three-dimensional $\mathrm{Ho}$ (II) coordination polymer based on $5^{\prime}$ -

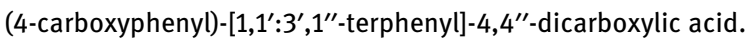
Inorg. Nano-Met. Chem. 2017, 12, 1746-1749.

5. Yao L. P., Zhao Y. Crystal structure of poly[diaqua- $\mu 5-4-$ (3,5-dicarboxylato $\mathrm{k}^{3} \mathrm{O}^{1}: O^{2}: O^{2}$-phenoxy) phthalato- $\mathrm{k}^{3} \mathrm{O}^{5}, \mathrm{O}^{7}: \mathrm{O}^{8}(\mu 2$ 4-(1H-pyrazol-5-yl)pyridine- $\left.{ }^{2} N: N^{\prime}\right) \operatorname{dicobalt(II)].~Z.~Kristallogr.~}$ NCS 2016, 231, 17-19.

6. Li S. H., Zhao Y. Synthesis and crystal structure of a new threedimensional Co(II) coordination polymer based on 3,3',4,5'-biphenyl tetracarboxylic acid and 4,4'-bipyridine. Inorg. Nano-Met. Chem. 2017, 47, 256-259.

7. Song W., Xu X. L., Yang Y. J., Li S. H. Crystal structure of catenapoly[( $\mu 3-5$-carboxy-2-(pyridin-4-yl)benzoato- $\left.{ }^{5} O, O^{\prime}: O^{\prime \prime}, O^{\prime \prime \prime}: O^{\prime \prime \prime}\right)$ (1,10-phenanthroline- $\left.\mathrm{K}^{2} N, N^{\prime}\right)$ cadmium(II)]. Z. Kristallogr. NCS 2018, 233, 755-756.

8. Li S. H., Han M. L., Liu G. Z., Ma L. F., Wang L. Y. Guest-induced single-crystal-to-single-crystal transformations of a new 4-connected 3D cadmium(II) metal-organic framework. RSC Adv. 2015, 5, 17588-17591.

9. Yao L. P., Zhao Y. Crystal structure of 4,4'-bipyridin-1-ium 3,3',5'-tricarboxy-[1,10-biphenyl]-2-carboxylate. Z. Kristallogr. NCS 2018, 233, 51-53.

10. Song W., Li S. H., Xu X. L. Crystal structure of 4-(4'-(pyridin-4-yl)[1,1'-biphenyl]-4-yl)pyridin-1-ium catena-poly[\{5-carboxy$4^{\prime}$-methyl-[1,1'-biphenyl]-3-carboxylato- $\left.\mathrm{K}^{2} O, O^{\prime}\right\}$ - $\left(\mathrm{K}^{2}-4^{\prime}\right.$-methyl[1,1'-biphenyl]-3,5-dicarboxylato-k40,0'"'')lead(II)]. Z. Kristallogr. NCS 2018, 233, 51-53. 